\title{
GAP-Independent Termination of Photoreceptor Light Response by Excess $\gamma$ Subunit of the cGMP-Phosphodiesterase
}

\author{
Steven H. Tsang, ${ }^{1}$ Michael L. Woodruff, ${ }^{2}$ Ching-Kang Chen, ${ }^{3}$ Clyde Y. Yamashita, ${ }^{4}$ Marianne C. Cilluffo, ${ }^{2}$ Anjali L. Rao, ${ }^{3}$ \\ Debora B. Farber, ${ }^{4}$ and Gordon L. Fain ${ }^{2,4}$ \\ ${ }^{1}$ Brown Glaucoma Laboratory, Department of Pathology, Center for Neurobiology and Behavior, College of Physicians and Surgeons, Columbia University, \\ New York, New York 10032, ${ }^{2}$ Department of Physiological Science, University of California Los Angeles (UCLA), Los Angeles, California 90095-1606, \\ ${ }^{3}$ Department of Biochemistry, Virginia Commonwealth University, Richmond, Virginia 23298-0614, and ${ }^{4}$ Jules Stein Eye Institute, UCLA School of \\ Medicine, Los Angeles, California 90095-7000
}

We have generated a mouse with rod photoreceptors overexpressing the $\gamma$ inhibitory subunit (PDE6 $\gamma$ ) of the photoreceptor G-protein effector cGMP phosphodiesterase (PDE6). PDE6 $\gamma$ overexpression decreases the rate of rise of the rod response at dim intensities, indicating a reduction in the gain of transduction that may be the result of cytoplasmic PDE6 $\gamma$ binding to activated transducin $\alpha$ GTP ( $\mathrm{T}_{\alpha}$-GTP) before the $\mathrm{T}_{\alpha}$-GTP binds to endogenous PDE6 $\gamma$. Excess PDE6 $\gamma$ also produces a marked acceleration in the falling phase of the light response and more rapid recovery of sensitivity and circulating current after prolonged light exposure. These effects are not mediated by accelerating GTP hydrolysis through the GAP (GTPase activating protein) complex, because the decay of the light response is also accelerated in rods that overexpress PDE6 $\gamma$ but lack RGS9. Our results show that the PDE6 $\gamma$ binding sites of PDE6 $\alpha$ and $\beta$ are accessible to excess (presumably cytoplasmic) PDE6 $\gamma$ in the light, once endogenous PDE6 $\gamma$ has been displaced from its binding site by $\mathrm{T}_{\alpha}$-GTP. They also suggest that in the presence of $\mathrm{T}_{\alpha}-\mathrm{GTP}$, the PDE6 $\gamma$ remains attached to the rest of the PDE6 molecule, but after conversion of $\mathrm{T}_{\alpha}$-GTP to $\mathrm{T}_{\alpha}$-GDP, the PDE6 $\gamma$ may dissociate from the PDE6 and exchange with a cytoplasmic pool. This pool may exist even in wild-type rods and may explain the decay of rod photoresponses in the presence of nonhydrolyzable analogs of GTP.

Key words: rod; phototransduction; retina; phosphodiesterase; G-protein; RGS protein

\section{Introduction}

Photoexcited rhodopsin in a vertebrate rod binds to and activates the G-protein transducin, facilitating the exchange of GTP for GDP on the transducin $\alpha$ subunit ( $\mathrm{T}_{\alpha}$ or GNAT1). The $\mathrm{T}_{\alpha}-\mathrm{GTP}$ then binds to the inhibitory $\gamma$ subunit (PDE6 $\gamma$ ) of the phosphodiesterase effector enzyme (PDE6), relieving the inhibition of the PDE6 $\alpha$ and $\beta$ catalytic subunits. Activated PDE6 hydrolyzes cGMP, leading to the closing of the cGMP-gated channels in the outer segment. This produces the hyperpolarizing light response

\footnotetext{
Received Nov. 7, 2005; revised March 13, 2006; accepted March 16, 2006.

This work was supported by the Foundation Fighting Blindness, the Burroughs-Wellcome Fund, the Hirschl Trust, the Dennis W. Jahnigen Award of the American Geriatrics Society, the Eye Surgery Fund of the Columbia College of Physicians and Surgeons, the Association of University Professors in Ophthalmology Research to Prevent Blindness, and National Institutes of Health Grants K08-EY004081 (S.H.T.), EY13811 (C.-K.C.), EY02651 (D.B.F.), and EY01844 (G.L.F.). We greatly appreciate the assistance of Marie E. Burns for her participation in preliminary experiments, and we thank her and Theodore $G$. Wensel for carefully reading this manuscript and providing many helpful comments. We are also grateful to Stephen P. Goff, Akio Yamazaki, Bernard K.-K. Fung, Frank D. Costantini, and members of their laboratories for the sharing of ideas, cell lines, antisera, and equipment; to Margery J. Fain for drawing supplemental Figure 4; and to the Helen RiaboffWhiteley Center (for G.L.F.), where some of the writing of this paper was done. We also thank the members of the Farber, Goff, and Gouras laboratories for initial support of these studies, especially to Won-Ho Lee, Chyuan Sheng Lin, and Jun Lin.

Correspondence should be addressed to Prof. Gordon L. Fain, Department of Physiological Science, University of California Los Angeles, 3836 Life Sciences, Los Angeles, CA 90095-1606. E-mail: gfain@ucla.edu. D01:10.1523/JNEUROSCI.4775-05.2006

Copyright $\odot 2006$ Society for Neuroscience $\quad$ 0270-6474/06/264472-09\$15.00/0
}

that signals the detection of the light to the rest of the nervous system (Fain, 2003).

The turnoff of the photoreceptor response and reopening of the channels requires the inactivation of rhodopsin by phosphorylation and subsequent binding of arrestin, as well as the return of the PDE6 to its dark resting level by hydrolysis of $\mathrm{T}_{\alpha}$-GTP back to $\mathrm{T}_{\alpha}$-GDP. The intrinsic rate of transducin GTP hydrolysis is slow (Antonny et al., 1993) but is facilitated by interaction of transducin with other proteins (Arshavsky et al., 2002). The first of these to be identified was PDE6 $\gamma$, which was initially thought to act by itself to accelerate GTP hydrolysis (Arshavsky and Bownds, 1992) but was later shown to have no effect on the rate of hydrolysis in isolation (Angleson and Wensel, 1993; Antonny et al., 1993) and to require additional components, subsequently identified as RGS9-1 (He et al., 1998), Gß5 (Makino et al., 1999), and a membrane anchor protein, R9AP (Hu and Wensel, 2002). These together form a GTPase activating protein (GAP) complex that is essential for the rapid conversion of $\mathrm{T}_{\alpha}$-GTP to $\mathrm{T}_{\alpha}$-GDP. The GAP complex proteins function in concert, because elimination of any one greatly reduces the rate of GTP hydrolysis and slows the rate of turnoff of the rod response (Chen et al., 2000; Krispel et al., 2003; Keresztes et al., 2004). The PDE6 $\gamma$ enhances the activity of the GAP complex probably by increasing the affinity of transducin for RGS9/G $\beta 5$ (Skiba et al., 2000), and disruption of 
this enhancement by mutation of the PDE6 $\gamma$ also slows photoreceptor response turnoff (Tsang et al., 1998).

The PDE6 $\gamma$ may also have an additional role in the inactivation of the photoreceptor light response. Several studies have shown that added PDE6 $\gamma$ can reduce the activity of PDE6 by a mechanism that is independent of the hydrolysis of $\mathrm{T}_{\alpha}$-GTP (Wensel and Stryer, 1990; Erickson et al., 1992; Angleson and Wensel, 1993, 1994; Otto-Bruc et al., 1993; Yamazaki, 1992; Yamazaki et al., 2002). We have re-examined this phenomenon by using genetic techniques to overexpress PDE6 $\gamma$ in mouse rods. Our experiments show that excess PDE6 $\gamma$ slows the rate of rise and decreases both the gain and sensitivity of the response; it also accelerates turnoff even in the absence of the GAP complex, probably by direct binding of the PDE6 $\gamma$ to the activated catalytic PDE6 $\alpha$ and $\beta$ subunits. These results were presented previously at a meeting of the Association for Research in Vision and Ophthalmology (Tsang et al., 2004).

\section{Materials and Methods}

Generation of mutant mouse lines. Mice were raised in normal room lighting ( $12 \mathrm{~h}$ on/off) and used in accordance with the Statement for the Use of Animals in Ophthalmic and Vision Research of the Association for Research in Vision and Ophthalmology, as well as the Policy for the Use of Animals in Neuroscience Research of the Society for Neuroscience. The ages of the animals ranged from 6 weeks to 1 year with no apparent difference in the results. DNA constructs for the expression of PDE6 $\gamma$ contained $4.4 \mathrm{~kb}$ of the mouse opsin promoter, the complete open reading frame of the PDE6 $\gamma \mathrm{cDNA}$ (Pde6g), and the polyadenylation signal of the mouse protamine gene (Lem et al., 1991). The entire Pde6g coding region in the transgenic construct was sequence verified. $K p n \mathrm{I}$ and $\mathrm{Xba \textrm {I }}$ were used to excise vector sequences from the construct.

Oocytes were obtained from super-ovulated F1 $(\mathrm{DBA} \times \mathrm{C} 57 \mathrm{BL} / 6) \mathrm{fe}-$ males mated with $\mathrm{F} 1(\mathrm{DBA} \times \mathrm{C} 57 \mathrm{BL} / 6)$. The construct was injected into the male pronuclei of oocytes under a depression slide chamber. These microinjected oocytes were cultured overnight in M16 and transferred into the oviducts of $0.5 \mathrm{~d}$ post coitum pseudopregnant F1 females. To limit the potential variation caused by differences in genetic background, all founders were backcrossed with the C57BL/6 strain. All mice were tested for the absence of the $r d 1$ mutation (Pittler and Baehr, 1991). To generate mice without the GAP complex, transgenic lines expressing the wt6C were crossed with the $R g s 9^{t m 1} / R g s 9^{t m 1}$ to place the transgene into RGS9-1 knock-out $\left(R g s 9^{-1-}\right)$ background.

Identification of transgenic mice. DNA was isolated from tail tips or liver samples by homogenizing the tissue, digesting extensively with proteinase $\mathrm{K}$, and extracting with phenol. Genotyping was determined by analyzing the $400 \mathrm{bp}$ PCR products generated by forward primer CTGGAATGGAAGGCCTGG and reverse primer ATGGTGTATGAGCGGCG. The DNAs were also analyzed by Southern blot hybridization with a Pde6g probe. Additional restriction digests were performed to analyze the structure of the integrated sequences and to insure that the DNA flanking the transgene was intact.

Rod outer-segment isolation. Rod outer segments (ROS) from darkadapted mice were isolated under dim red light in a HEPES/phosphatebuffered balanced salt solution containing the following (in $\mathrm{mM}$ ): 4.09 $\mathrm{NaH}_{2} \mathrm{PO}_{4}, 148.4 \mathrm{NaCl}, 4.91 \mathrm{KCl}, 2.45 \mathrm{CaCl}_{2}, 1.23 \mathrm{MgSO}_{4}$, and 14.7 HEPES, pH 7.2 (Zimmerman and Godchaux, 1982). Rhodopsin content was determined by the difference in absorbance at $500 \mathrm{~nm}$ before and after bleaching.

Immunoblot analyses. To facilitate the quantification of transgenic PDE6 $\gamma$, transgenic mice were backcrossed toPde $6 g^{t m 1} / P d e 6 g^{t m 1}$ to obtain mice that expressed only the transgenic form of PDE6 $\gamma$ (Tsang et al., 1996). Mouse retinas were homogenized in PBS. Protein concentration was determined against BSA using the BCA kit (Pierce, Rockford, IL). The indicated amounts of retinal extracts were resolved by SDS-PAGE and transblotted onto nitrocellulose membrane. The relative levels of retinal proteins were determined by immunoblotting and enhanced chemiluminescence (ECL) with primary antibodies against RGS9-1

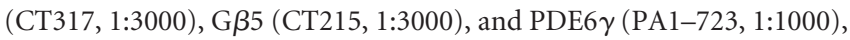
followed by peroxidase-conjugated secondary goat anti-rabbit IgG (1: 5000; Santa Cruz Biotechnology, Santa Cruz, CA). ECL signals were captured by a Kodak (Rochester, NY) IS440 imaging station and quantified with Kodak 1D4.5 software.

To detect PDE6 $\alpha$ and $\beta$ subunits, protein containing 185 pmol of rhodopsin/lane from murine ROS was separated by electrophoresis on a 6.5-9.5\% acrylamide/ $1.5 \%$ crosslinker inverted gradient gel, as described previously (Tsang et al., 1996). Proteins were then transferred to $0.2 \mathrm{~mm}$ immunoblot polyvinylidene difluoride membranes (Bio-Rad, Hercules, CA) and left overnight at $4 \mathrm{~V} / \mathrm{cm}$ by the method of Towbin et al. (1979). Membranes were blocked in 3\% BSA in $500 \mathrm{~mm} \mathrm{NaCl}, 20 \mathrm{~mm}$ Tris, $\mathrm{pH}$ 7.6, and $0.1 \%$ Tween 20 . The PDE6 $\alpha$ and $\beta$ subunits were detected by incubation with a polyclonal antiserum raised against a 17 -mer peptide (Piriev et al., 1993), 100\% homologous with the rod PDE6 $\alpha$ and $\beta$ subunits as well as with the cone PDE $\alpha$ subunit. Western blots were visualized with the DuoLux Chemiluminescence substrate kit (Vector Laboratories, Burlingame, CA) and a goat anti-rabbit IgG-alkaline phosphatase conjugate. Blots were exposed to Hyperfilm-MP (Amersham Biosciences, Piscataway, NJ) preflashed to increase sensitivity and linearity according to the SensitizeJ protocol (Amersham Biosciences). Signals were quantified by densitometric scanning.

Similar methods were used to assess relative levels of other retinal proteins (supplemental Fig. 1, www.jneurosci.org as supplemental material), by immunoblotting and ECL with primary antibodies against RGS9-1 (CT317, 1:3000), G 35 (CT215, 1:3000), PDE6 $\gamma$ (PA1-723, 1:1000; Affinity Bioreagents, Golden, CO), GC-E (K285, 1:5000; from D. Garbers, University of Texas Southwestern Medical Center at Dallas, Dallas, TX), GC-F (A670, 1:5000; from D. Garbers), PDE6 $\alpha$ (PA1-720, 1:500; Affinity Bioreagents), RK (MA1-721, 1:5000; Affinity Bioreagents), arrestin (Marr, 1:20,000), transducin $\alpha$ subunit (UUTA1, 1:5000), phosducin (Gerti, 1:5000; from R. Lee, Sepulveda Veterans Affairs Hospital, Los Angeles, CA), GCAP1 ( $\alpha$-GCAP1, 1:1000; from A. Dizhoor, Pennsylvania College of Optometry, Elkins Park, PA), GCAP2 ( $\alpha$-GCAP2, 1:1000; from A. Dizhoor), followed by peroxidaseconjugated secondary goat anti-rabbit or goat anti-mouse IgG (1:5000; Santa Cruz Biotechnology).

Histology and immunocytochemistry. Mice were deeply anesthetized with pentobarbital $\left(100 \mathrm{mg} \mathrm{kg}^{-1}\right)$ and fixed by vascular perfusion for 5 min with $2.5 \%$ glutaraldehyde and $2 \%$ paraformaldehyde in $0.1 \mathrm{M}$ phosphate buffer, $\mathrm{pH}$ 7.3. Eyes were enucleated and a section of cornea removed. The eyes were again immersed in fixative for $2 \mathrm{~h}$. The lens was removed, and the eyes were left in fixative overnight. The eyeballs were postfixed in osmium tetroxide, dehydrated through a graded series of ethanol, and bisected through the optic nerve. Each half was then embedded in Epon (Eponate 812; Ted Pella, Redding, CA). For light microscopy (supplemental Fig. 2, available at www.jneurosci.org as supplemental material), sections $(1 \mu \mathrm{m})$ were cut, mounted on glass slides, and stained with toluidine blue. Similar areas were examined from each eye, as determined by measurement from the optic nerve head. For analysis of the outer segment diameters, silver-gold sections $(60-90 \mathrm{~nm})$ were cut on an ultramicrotome, picked up on grids, and stained with uranyl acetate and lead citrate. Sections were photographed, the negatives were scanned, and the average outer segment diameter was determined from each of 18 outer segments from both wild-type (WT) and wt6C retinas. The means for each eye were then averaged.

For immunohistochemistry, mouse eyeballs were fixed in $4 \%$ paraformaldehyde in PBS at $4^{\circ} \mathrm{C}$ overnight and cryoprotected in $30 \%$ sucrose in PBS. The eyeballs were sectioned at $12 \mu \mathrm{m}$ thickness, washed twice with PBS, and blocked with $10 \%$ goat serum in $0.3 \%$ Triton X-100 in PBS for $30 \mathrm{~min}$. They were then incubated with primary antibodies overnight at the following concentrations: PDE6 $\gamma$ (PA1-723), 1:100; GNAT1 (UUTA1), 1:100; arrestin (Marr), 1:200. Sections were washed three times for 5 min with PBS and treated with FITC-conjugated goat antirabbit $\operatorname{IgG}(1: 100$; Southern Biotechnology, Birmingham, AL) for $1 \mathrm{~h}$ at room temperature. After washing with PBS three times for $10 \mathrm{~min}$, they were coverslipped with Vectashield and examined under a Leitz (Wetzlar, Germany) fluorescent microscope. Fluorescent images were cap- 
tured with a SpotMosaic digital camera and exported to and edited with Microsoft (Redmond, WA) PowerPoint software.

Suction electrode measurements. Methods for recording responses of mouse rods have been given previously (Woodruff et al., 2002, 2003). In brief, rods were perfused with physiological solution containing amino acids and nutrients kept at $37^{\circ} \mathrm{C}$. Stimuli at $500 \mathrm{~nm}$ were attenuated with calibrated absorptive neutral-density filters. Responses were amplified by a Warner Instruments (Hamden, CT) patch-clamp amplifier (PC-501A) and recorded with pClamp hardware and software (Molecular Devices, Foster City, CA). The single-photon response was calculated from the squared mean and variance (Chen et al., 2000). All errors given here (including in the table and figures) are SE.

\section{Results}

To understand in greater detail the role of PDE6 $\gamma$ in photoreceptor response turnoff, we overexpressed PDE6 $\gamma$ in mouse rods. Germ-line transmission was obtained from nine founders, which showed varying levels of PDE6 $\gamma$ expression compared with WT C57BL/6 controls (Fig. $1 A)$. No PDE6 $\gamma$ was detected either in postnatal day 13 (P13) (Fig. 1A) or adult Pdeg ${ }^{\mathrm{tm} 1} /$ Pdeg $^{\mathrm{tm} 1}$ mice (Tsang et al., 1996), in which the PDE6 $\gamma$ gene had been knocked out. The line wt6C, which from densitometric scanning we determined to express a level of PDE6 $\gamma$ protein twofold higher than in WT animals, was selected for additional experimentation. Immunoblot analyses showed that the levels of other components of the RGS complex, RGS9-1 and G $\beta 5$, were normal in these animals (Fig. $1 B$ ), as well as the catalytic subunits of PDE6 $\alpha$ and PDE6 $\beta$ (Fig. 1C). This was also true of other proteins involved in phototransduction cascade (supplemental Fig. 1, available at www. jneurosci.org as supplemental material), including rhodopsin kinase, arrestin, the GCAPs, and guanylyl cyclase (both E and F). Thus, the PDE6 $\gamma$ overexpression appeared to have no effect on the expression of other photoreceptor proteins important in the transduction cascade. The effect on transduction (see below) can thus be attributed solely to the increase in PDE6 $\gamma$ level.

Immunohistochemical experiments showed that PDE6 $\gamma$ in both WT and wt6C animals was localized to the ROSs and that the distribution of PDE6 $\gamma$ was the same in the light and in the dark (Fig. 2). This is in contrast to the localization of $\mathrm{T}_{\alpha}$ and arrestin. As in previous studies (Brann and Cohen, 1987; Philp et al., 1987; Whelan and McGinnis, 1988; Sokolov et al., 2002; Zhang et al., 2003), the $\mathrm{T}_{\alpha}$ in the dark in both WT (data not shown) and wt6C retinas is mostly concentrated in the outer segments of the rods and moves in the light to the inner segments. Arrestin, on the other hand, is abundant in the inner segments in the dark but is found in both WT (data not shown) and wt6C retinas almost exclusively in the outer segments in the light (Philp et al., 1987; Whelan and McGinnis, 1988; Mendez et al., 2003; Zhang et al., 2003; Nair et al., 2005). These experiments provide no indication that the PDE6 $\gamma$ of wt6C animals and the other components of the transduction cascade behave in any way dif- ferent from those in WT mice. Furthermore, as Figure 2 shows (see also supplemental Fig. 2, available at www.jneurosci.org as supplemental material), the morphology of wt6C mouse retina was normal, and the amplitude of the a-wave of the electroretinogram of wt6C mice was also normal up to 9 months of age (data not shown). The diameter of the ROSs was also the same in wt6C mice as in wild type $(\sim 1.3 \mu \mathrm{m})$.

\section{Rod responses in retinas with overexpressed PDE6 $\gamma$}

Representative responses of a WT rod and a wt6C rod to flashes of light of increasing intensity are given in Figure 3, $A$ and $B$. Although rods in both animals have approximately the same average dark current (Table 1) and respond over a wide range of light levels, the responses of wt6C rods were desensitized, as can be seen from the mean response-intensity curves in Figure $3 C$. They also rise more slowly, especially at low intensities, and decay with an accelerated time course. A similar effect on sensitivity (although apparently not on response waveform) was seen after introduction of a much smaller concentration of excess PDE6 $\gamma$ into toad rods (Rieke and Baylor, 1996).

We examine the differences in waveform in greater detail in Figure $4 A-C$. Here, we have superimposed averaged responses from many rods of WT (black traces) and wt6C (red traces) mice at three different light intensities. The responses for each cell have been normalized before averaging to the maximum value of the 


\section{Dark}
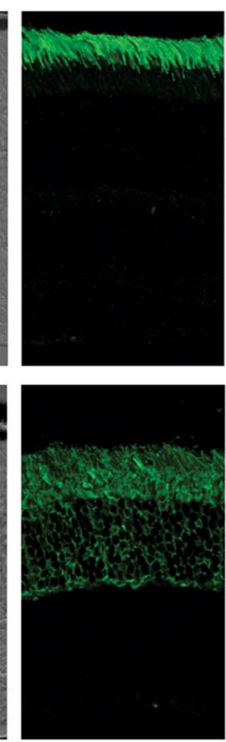

Arr
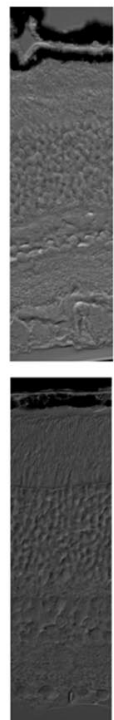

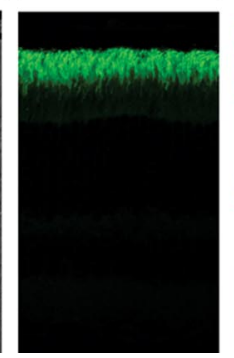

Light
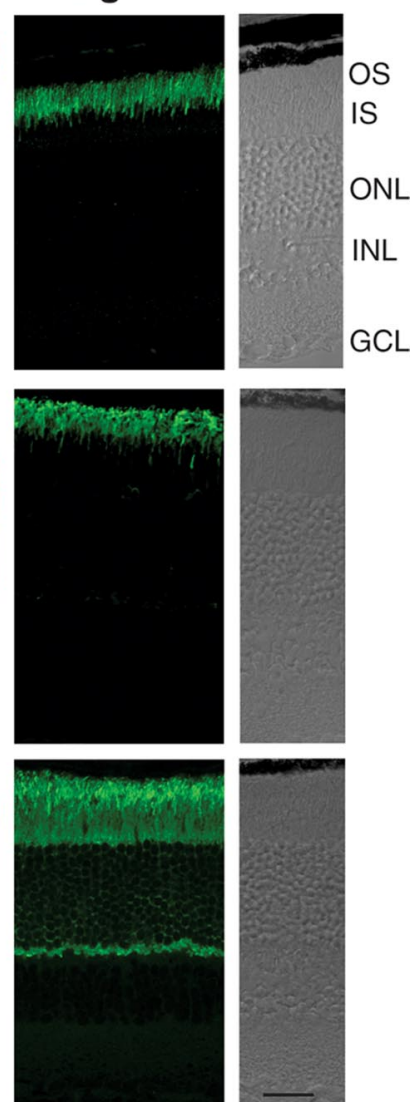

Figure 2. Absence of light-dependent movement of PDE6 $\gamma$ in wt6C transgenic mice. Fluorescent and bright-field images of retinal localization of PDE6 $\gamma$ (PDE $\gamma$; top panels), arrestin (Arr; middle panels), and transducin $\alpha$ subunit (T $\alpha$; bottom panels) in dark-adapted and lightadapted conditions were examined by immunohistochemistry (see Materials and Methods). For the light-adapted condition, mice were exposed to continuous room light (60 lux) for $6 \mathrm{~h}$; for the dark-adapted condition, mice were killed after $12 \mathrm{~h}$ in darkness under infrared illumination. Although $\mathrm{T} \alpha$ and arrestin redistribute in opposite directions in and out of the outer segment (OS) layer, PDE6 $\gamma$ is found exclusively in the OS regardless of light. IS, Inner segment; ONL, outer nuclear layer; INL, inner nuclear layer; $\mathrm{GCL}$, ganglion cell layer. Scale bar, $25 \mu \mathrm{m}$.

photo current for that cell. The insets compare the initial time course of the response at a higher temporal resolution.

At the dimmest intensity (Fig. $4 \mathrm{~A}$, inset), there is a clear difference in the initial rising phase of the light response that is manifest even at early times. This difference represents a reduction in the gain of the transduction cascade in the wt6C rods. It is also present at a moderate light intensity (Fig. $4 B$, inset), although in bright light (Fig. $4 C$, inset), the difference in rise time nearly disappears. If this difference is the result of excess PDE6 $\gamma$ in wt6C rods binding to $\mathrm{T}_{\alpha}$-GTP before it can bind to endogenous PDE6 $\gamma$ (Wensel and Stryer, 1990; Otto-Bruc et al., 1993), the results in Figure $4 A-C$ indicate that the amount of PDE6 $\gamma$ is sufficient to alter the gain at dim to moderate intensities but that at bright intensities the $\mathrm{T}_{\alpha}$-GTP is so abundant in proportion to PDE6 $\gamma$ that the gain of transduction is virtually unaffected.

The results in Figure $4 A-C$ also show that the responses of wt6C rods decay more rapidly than those of WT rods, and this is apparent even at the dimmest intensity (Fig. 4A). At bright intensities (Fig. 4C), the waveforms of decay become similar in appearance but are shifted along the time axis. We constructed a Pepperberg plot (Pepperberg et al., 1992) for the WT and wt6C responses to bright flashes, by plotting the time for decay $\left(\mathrm{T}_{\text {sat }}\right)$ of
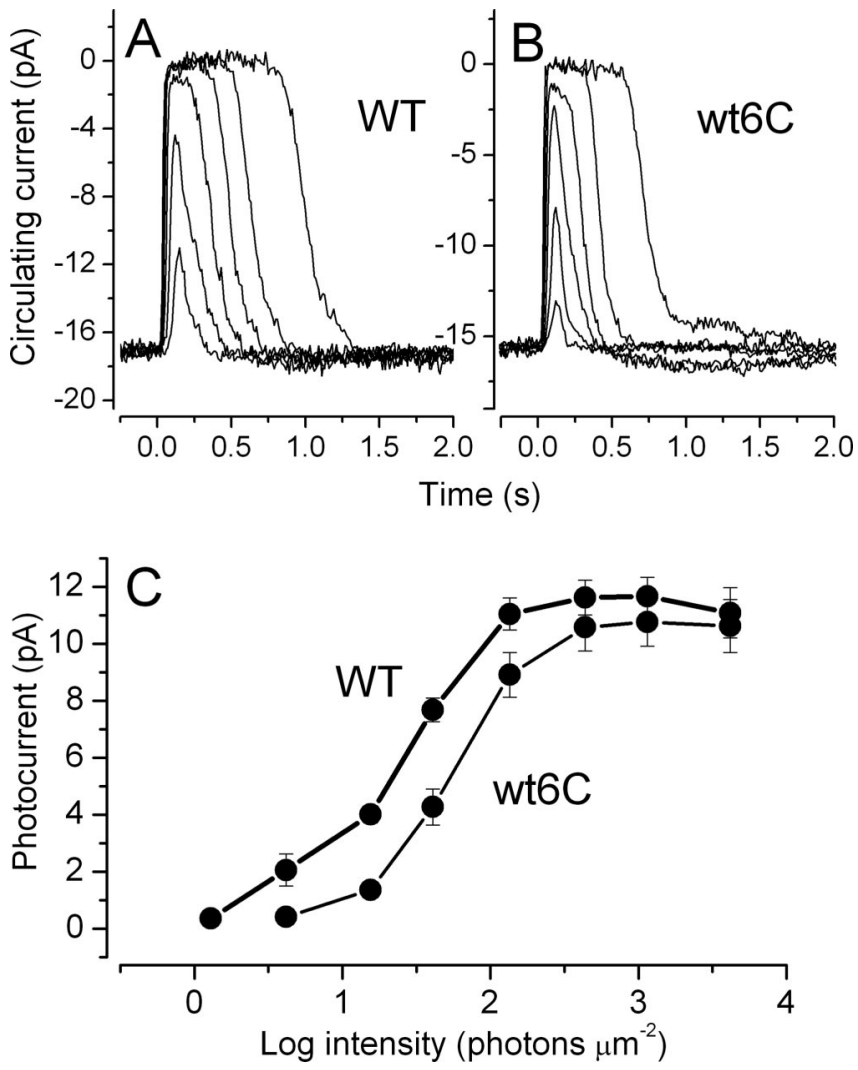

Figure 3. Responses of wt $6 C$ and WT rods to light. $A, W T$ rod, 20 ms flashes of $500 \mathrm{~nm}$ light at flash intensities of $17,43,160,450,1120$, and 4230 photons $\mu \mathrm{m}^{-2}$. The traces are averages of two to four flashes at each intensity. $\boldsymbol{B}$, Typical responses from a wt $6 \mathrm{C}$ rod to the same flash intensities. Each trace was averaged from three to eight flashes. $\boldsymbol{C}$, Response amplitude versus flash intensity averaged from $34 \mathrm{WT}$ and $20 \mathrm{wt} 6 \mathrm{C}$ rods. Flash intensity required to elicit a response of half-maximal amplitude was shifted to higher intensities by $\sim 0.3 \log$ units in the wt6C rods (see Table 1).

the response to a criterion level of $25 \%$ of the dark current (or $75 \%$ of the maximum response), as a function of the natural log of the light intensity (Fig. 4D). We found that the curves for WT and wt6C rods had a similar shape but were shifted along the time axis. The curves also seem slightly different in slope, indicating a different value for the dominant time constant in response decay $T_{\mathrm{d}}$. We investigated this possibility in greater detail by calculating the value of $T_{\mathrm{d}}$ for each rod separately and then averaging. This gave $173 \pm 8 \mathrm{~ms}$ for WT $(n=44)$ and $147 \pm 10 \mathrm{~ms}$ for wt6C $(n=$ 27). A Student's $t$ test gave a $p$ value for this difference of 0.052 , indicating that the difference in $T_{\mathrm{d}}$ is just above the 0.05 criterion for significance.

Figure 5 gives the mean single-photon responses of rods from the WT and wt6C animal strains, calculated as previously (see Materials and Methods) (Woodruff et al., 2003). Once again, the smaller and more rapidly decaying response is the one from the wt6C animals. Like the dim-intensity responses in Figure $4 A$, the rise time of the wt6C single-photon response is slower than that of WT rods (Fig. 5, inset), indicating a difference in the gain of transduction. There is also a marked acceleration in the decay of the single-photon response, demonstrating that the effect of excess PDE6 $\gamma$ on response turnoff occurs even at the limit of the smallest response.

We compare some parameters of the responses of rods from WT and wt6C mice in Table 1 . The time to peak of wt6C rods was smaller than that of WT rods, and this difference was significant ( $t$ test; $p<0.05$ ). The sensitivity of wt6C rods was smaller by a 
Table 1. Kinetic and sensitivity parameters of WT and wt $6 \mathrm{C} \mathrm{rods}$

\begin{tabular}{lllllcc}
\hline & $\begin{array}{l}\text { Dark current } \\
(\mathrm{pA})\end{array}$ & $\begin{array}{l}\text { Time to peak } \\
(\mathrm{ms})^{a}\end{array}$ & $\begin{array}{l}\text { Flash sensitivity } \\
\left(\mathrm{pA} \mathrm{photon}{ }^{-1} \mu \mathrm{m}^{-2}\right)\end{array}$ & $\begin{array}{l}\text { Flash intensity at half-saturation } \\
\left(\text { photons } \mu \mathrm{m}^{-2}\right)\end{array}$ & $\begin{array}{l}\text { Integration time } \\
(\mathrm{ms})^{a}\end{array}$ & $\begin{array}{c}\tau \text { for response recovery } \\
(\mathrm{ms})^{b}\end{array}$ \\
\hline WT & $11.9 \pm 0.5(44)$ & $143 \pm 4(46)$ & $0.26 \pm 0.02(46)$ & $31 \pm 2(43)$ & $199 \pm 20(47)$ & $205 \pm 19(45)$ \\
wt6C & $11.1 \pm 0.7(29)$ & $126 \pm 6(29)$ & $0.10 \pm 0.01(29)$ & $71 \pm 6(29)$ & $103 \pm 9(29)$ & $91 \pm 11(29)$ \\
Rgs9 ${ }^{-/-}$ & $8.2 \pm 0.7(15)$ & $214 \pm 18(19)$ & $0.15 \pm 0.02(20)$ & $31 \pm 3(15)$ & $1820 \pm 450(21)$ & $8420 \pm 1000(17)$ \\
Rgs9 $^{-/-}$/wt6C & $11.6 \pm 0.7(10)$ & $127 \pm 15(9)$ & $0.16 \pm 0.05(9)$ & $78 \pm 13(9)$ & $236 \pm 68(9)$ & $326 \pm 60(9)$ \\
\hline
\end{tabular}

Values are means \pm SE with the number of cells in parentheses.

${ }^{a}$ Values are for light responses at intensity of 15.5 photons $\mu \mathrm{m}^{-2}$.

${ }^{b}$ Single exponential fit of just-saturating response.

factor of 2-3, and the flash intensity required to produce a half-saturation response was larger by approximately a factor of 2 (or $0.3 \log _{10}$ units) (Fig. 3C); these differences were again significant $(t$ test; $p<0.05)$. The integration time and time constant for response recovery are both significantly shorter for wt6C rods ( $t$ test; $p<0.01)$, reflecting the more rapid time course of response decay. Thus, the more extensive data in Table 1 support the conclusions of Figures 3-5: that responses of rods in wt6C mice are desensitized and decay more rapidly than those of WT rods.

\section{GAP-independent acceleration of turnoff by overexpressed PDE6 $\gamma$} Because PDE6 $\gamma$ is known to facilitate the interaction of RGS9 with $\mathrm{T}_{\alpha}$, the results in Figure 3 and Table 1 do not distinguish between a direct effect of PDE6 $\gamma$ on PDE6 turnoff and an indirect effect mediated through the GAP complex. We therefore intercrossed wt6C mice into an $\mathrm{Rgs}^{-/-}$ background that lacks both the RGS9-1 and G $\beta 5 \mathrm{~L}$ proteins (Chen et al., 2000). $R g s 9^{-/-}$rods have prolonged light responses (Fig. 6A), as reported previously (Chen et al., 2000). In $\mathrm{Rgs}^{-/-}$rods expressing wt6C, however, the rate of decline of the rod response was greatly accelerated (Fig. 6B), although still much slower than in WT or wt6C rods. Note the differences in time scale between Figure $6 B$ and Figures $3, A$ and $B$, and $4 A-C$. Single exponential fits to the decay phase of just-saturating responses gave a time constant of $91 \pm 11 \mathrm{~ms}$ for wt6C rods and $205 \pm 19 \mathrm{~ms}$ for WT rods but $8.42 \pm 1.0 \mathrm{~s}$ for $R g s 9^{-1-}$ rods and $326 \pm 60 \mathrm{~ms}$ for rods from progeny of the mating of $\mathrm{Rgs}^{-1-}$ and wt6C mice. The integration time was also longer for rods from these animals than for those from either WT or wt6C rods (Table 1 ).

In $R g s 9^{-1-}$ rods, recovery after a bright flash is delayed. This can be seen in the recording of Figure $7 A$, in which a rod was exposed to a saturating light for $4 \mathrm{~min}$, ending at $t=0$. Flashes were not given during the recovery of the photo current, so as not to prolong return of the current to the baseline even further. In rods from $R g s 9^{-1-}$ mice mated to wt6C (Fig. $7 B$ ), the recovery was greatly accelerated and flashes during recovery produced photo currents of normal amplitude. Recovery in these rods was still considerably slower than in WT (Fig. 7C) or wt6C (data not shown) rods, for which the current returned to the baseline only a few seconds after the turning off of the background light. These results complement those of Figure 6 and show that the overexpression of PDE6 $\gamma$ can at least, to some extent, reverse the effects of knock-out of the $R g s 9$ gene (i.e., overexpression has an epistatic effect on rods lacking the GAP complex). We return to this observation in the Discussion.

\section{Continuous component of dark noise}

The experiments we have so far described indicate that excess PDE6 $\gamma$ can diffuse presumably from the cytoplasm to bind to the PDE6 $\gamma$ binding sites of the PDE6 $\alpha$ and $\beta$ catalytic subunits. For this to occur, the binding sites must be accessible to the excess PDE6 $\gamma$. We wondered whether these sites were also accessible in darkness. Rods in darkness exhibit two kinds of noise (Baylor et al., 1980, 1984), one consisting of discrete events resembling 


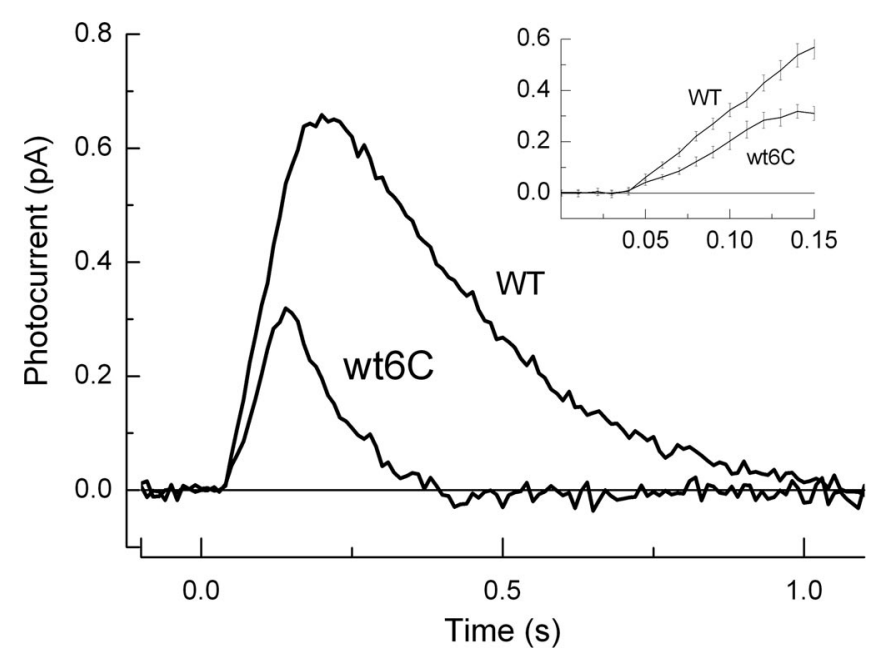

Figure 5. Averaged single-photon responses for WT and wt $6 \mathrm{C} \mathrm{rods} \mathrm{from} 47 \mathrm{WT}$ and $18 \mathrm{wt} 6 \mathrm{C}$ rods. Individual single-photon responses were calculated by dividing the mean response to 40 - 60 dim light-flash responses by the number of photoisomerizations per flash, which was estimated by the scaling factor needed to adjust the individual rising phases of the mean responses to the rising phases of the ensemble variances of the means. Inset, Rising phases of single-photon responses on an expanded time scale with the SE at $10 \mathrm{~ms}$ intervals.

single-photon responses and another continuous component, probably produced by spontaneous activity of PDE6 in the dark (Rieke and Baylor, 1996). We reasoned that if excess PDE6 $\gamma$ could bind to the PDE $\alpha \beta$ core in darkness, the wt $6 \mathrm{C}$ rods might have a smaller component of continuous noise than WT rods.

We therefore recorded baseline noise in segments of $10 \mathrm{~s} \mathrm{du}$ ration; 20-30 such segments were recorded from each rod both in the dark and in the presence of saturating light. From these recordings, we determined the power spectrum of the dark fluctuations for each cell by subtracting the spectrum in the light from the spectrum in the dark. These difference spectra were then averaged for a total of $17 \mathrm{WT}$ and 18 wt6C rods. We observed a clear light-dark difference in noise in both WT and wt6C rods, but we could detect no significant difference in the averaged spectra of the rods of the two kinds. We therefore conclude either that excess PDE6 $\gamma$ does not bind to the PDE6 $\gamma$ binding sites of the PDE6 $\alpha$ and $\beta$ catalytic subunits in darkness (Rieke and Baylor, 1996) or that the excess PDE6 $\gamma$ does bind and have an effect on the continuous component, but this effect was too small for us to observe.

\section{Discussion}

Our experiments indicate that excess PDE6 $\gamma$ can decrease the rate of rise and gain of the rod response. Comparisons of waveforms of single-photon (Fig. 5) and dim-flash (Fig. 4A) responses show that the responses of wt6C and $\mathrm{WT}$ rods diverge at a time too early to reflect accelerated turnoff of PDE6 (Tsang et al., 1998). The simplest explanation is that excess PDE6 $\gamma$ can bind to $\mathrm{T}_{\alpha^{-}}$ GTP in the outer segments of wt6C rods (Wensel and Stryer, 1990; Otto-Bruc et al., 1993), so that fewer $\mathrm{T}_{\alpha}$-GTPs are available to bind to endogenous PDE6 $\gamma$. The concentration of activatable transducin may also be reduced, if added PDE6 $\gamma$ were to bind to $\mathrm{T}_{\alpha}$-GDP and remove it from $\mathrm{T}_{\beta \gamma}$ (Otto-Bruc et al., 1993). The effects on rise time and gain are marked at dim intensities (Fig. $4 A$ ) but become less easily observed for flashes of increasing intensity (Fig. $4 B, C$ ), perhaps because the number of PDE6 $\gamma$ molecules in the cytoplasm is not large enough to produce a significant effect when large amounts of $\mathrm{T}_{\alpha}$-GTP are generated. A comparison of the number of molecules is revealing. For Figure 4,
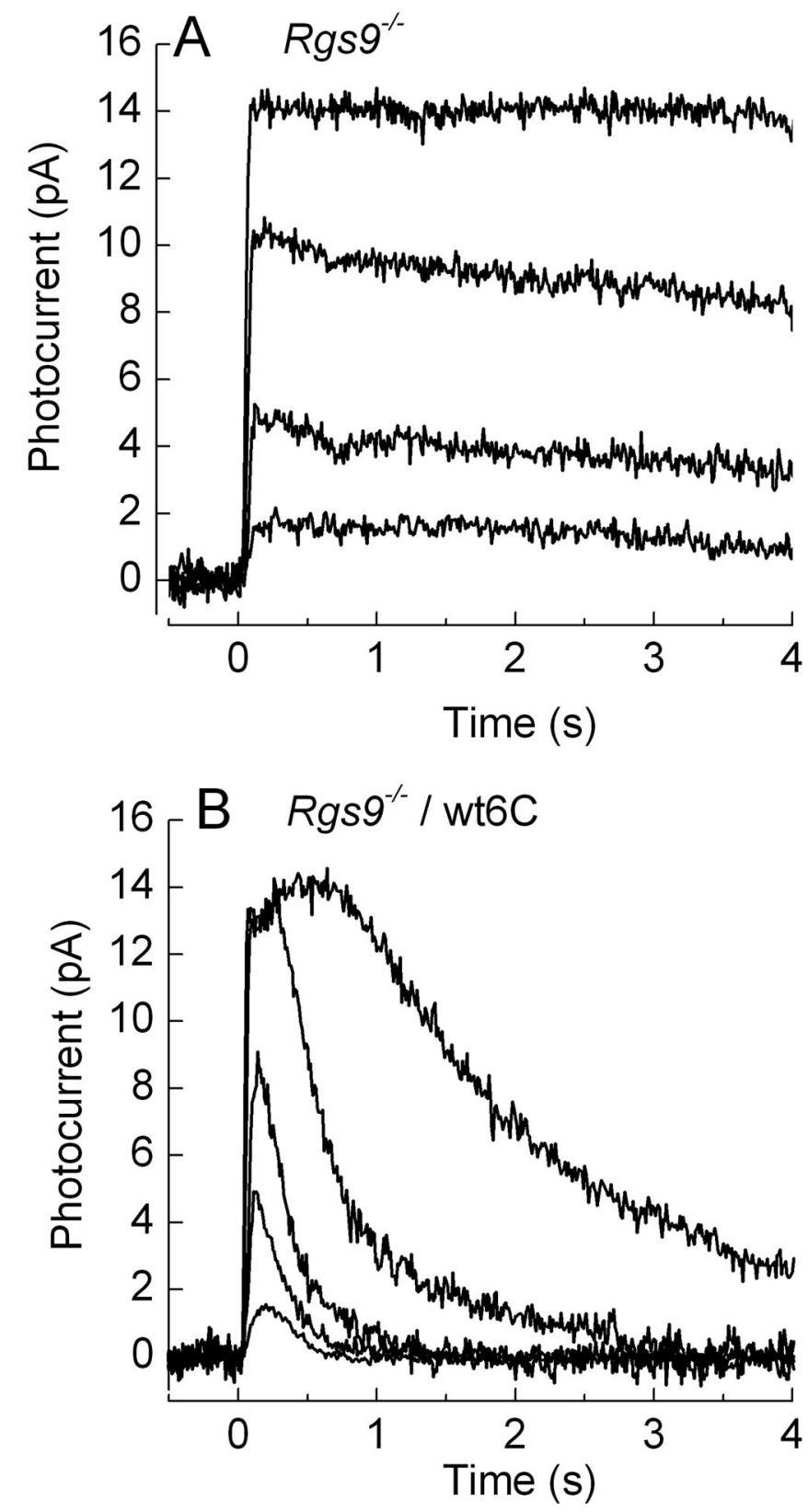

Figure 6. Responses of rods lacking the RGS9-1 complex are accelerated by excess PDE6 $\gamma$. $\boldsymbol{A}$, Responses of a $R g s 9^{-/-}$rod to flash intensities of $4,17,43$, and 160 photons $\mu \mathrm{m}^{-2}$. $\boldsymbol{B}$, Responses of rods from wt6C mice on an $R g s 9^{-/-}$background. See Results. Flash intensities are $4,17,43,160$, and 450 photons $\mu \mathrm{m}^{-2}$. The responses are averages of 5-20 flashes each.

$B$ and $C$, the flash intensities were 160 and 1120 photons $\mu \mathrm{m}^{-2}$, equivalent to $\sim 80$ and $560 \mathrm{Rh}^{*}$ per rod [assuming a collecting area of $0.5 \mu \mathrm{m}^{2}$ (Field and Rieke, 2002)]. If each bleached rhodopsin molecule produces $120 \mathrm{~T}_{\alpha}$-GTP molecules (Leskov et al., $2000)$, then, as a result of the two flashes, $9.6 \times 10^{3}$ and $6.7 \times 10^{4}$ $\mathrm{T}_{\alpha}$-GTP molecules will be formed. The number of excess $\gamma$ molecules in our wt6C animals, on the other hand, is of the same order as the number of endogenous $\gamma$ molecules, $\sim 30 \mu \mathrm{M}$ [twice the PDE concentration of $\sim 15 \mu \mathrm{M}$ (Hamm and Bownds, 1986)]. This is equivalent to $\sim 2 \times 10^{5}$ molecules, substantially greater than our best estimates of the number of $\mathrm{T}_{\alpha}$-GTP molecules produced by the flashes. Because the brighter of the two flashes showed little effect of excess PDE6 $\gamma$ on the rising phase of the response, there are three possible conclusions. The effective con- 


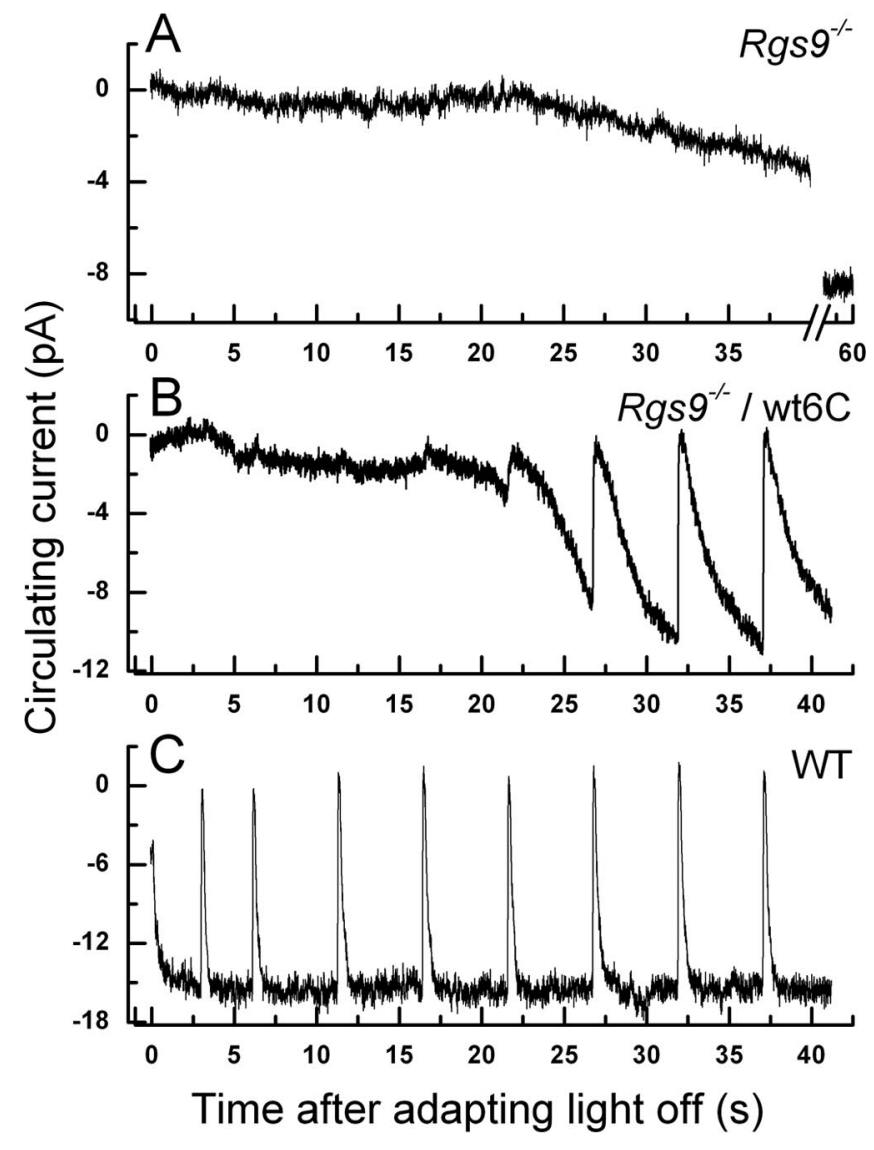

Figure 7. Recovery from bright-light exposure $\left(4 \mathrm{~min}, 2830\right.$ photons $\left.\mu \mathrm{m}^{-2} \mathrm{~s}^{-1}\right)$. The adapting light was turned off at $t=0$. The pigment bleached was $<0.4 \% . A, R g s 9^{-1-}$ rod. The break in the abscissa shows eventual recovery of $R g s 9^{-/}$rod response $50-60 \mathrm{~s}$ after the light was extinguished. $\boldsymbol{B}$, wt $6 \mathrm{C}$ rod on Rgs $9^{-/-}$background. Flashes of $20 \mathrm{~ms}$ at 437 photons $\mu \mathrm{m}^{-2}$ were presented every $5 \mathrm{~s}$ after turning off the adapting light. $\boldsymbol{C}$, WT rod with flashes as in $\boldsymbol{B}$.

centration of PDE6 $\gamma$ is much less than the total concentration, or estimates of the gain of production of $\mathrm{T}_{\alpha}$-GTP are too low by at least 1 order of magnitude, or $\mathrm{T}_{\alpha}$-GTP binds much more readily and tightly to PDE6 $\gamma$ on the membrane than to excess PDE6 $\gamma$, presumably in the cytoplasm.

Our results show that, in addition to its effect on the rising phase, excess PDE6 $\gamma$ also accelerates the rate of turnoff of the photoreceptor response, confirming for intact rods earlier biochemical observations on isolated proteins or ROS preparations (Wensel and Stryer, 1990; Erickson et al., 1992; Angleson and Wensel, 1993, 1994; Antonny et al., 1993; Bondarenko et al., 1999; Yamazaki et al., 2002). The effect of excess PDE6 $\gamma$ on response decay is not simply attributable to a decrease in PDE activation, because even for WT and wt6C responses with similar rising phases and producing a similar suppression of circulating current, the wt6C response decays markedly more rapidly (Fig. $4 C$ and supplemental Fig. 3). The acceleration of mouse rod response decline can occur even in the absence of RGS9-1 and the GAP complex, indicating that shutoff by excess PDE6 $\gamma$ is independent of any effect it may have on the hydrolysis of GTP.

The most likely interpretation of our experiments, in our view, is that the PDE6 is rate limiting for the decay of the rod photoreceptor light response (Sagoo and Lagnado,1997; Krispel et al., 2005) and that excess PDE6 $\gamma$ speeds up response decay by accelerating the rate of turnoff of PDE activity. One difficulty with this interpretation is that excess PDE6 $\gamma$ does not significantly alter the value of the rate-limiting time constant, $T_{\mathrm{d}}$ (Fig. $4 D)$. We think the reason for this is that the effective concentration of the overexpressed PDE6 $\gamma$ is sufficient to turn off only a fraction of the PDE6 activated by bright light and that the majority is extinguished in the usual way by GTP hydrolysis, as in a WT rod. Because, however, the mechanisms of response turnoff are complex and still not completely understood, other explanations cannot at present be excluded.

Although PDE6 $\gamma$ binding and shut down of the PDE6 can occur in the absence of the GAP complex, these proteins do have an effect on the rate of binding. This can be seen from the following considerations. In WT mice, the response to a just-saturating light decays with a mean time constant of $205 \mathrm{~ms}$ (Table 1). It is likely from our own results and those of Krispel et al. (2005) that this represents the time constant of $\mathrm{T}_{\alpha}$-GTP hydrolysis. In animals overexpressing PDE6 $\gamma$, the light response can be turned off in two ways, either by hydrolysis of $\mathrm{T}_{\alpha}$-GTP or by binding of free PDE6 $\gamma$. If these two processes occur independently, then their rate constants should add and the time constant of turnoff in animals overexpressing PDE6 $\gamma(91 \mathrm{~ms})$ should be the inverse of the sum of inverses of the time constants of $\mathrm{T}_{\alpha}-\mathrm{GTP}$ hydrolysis and of PDE6 $\gamma$ binding. The time constant for PDE6 $\gamma$ binding can therefore be calculated to be $\sim 164 \mathrm{~ms}$. If this same calculation is performed for rods lacking the GAP complex, the predicted time constant for PDE6 $\gamma$ binding is $339 \mathrm{~ms}$, over twice as long. The simplest explanation is that the GAP complex facilitates excess PDE6 $\gamma$ binding, perhaps by removing $\mathrm{T}_{\alpha}$-GTP/PDE6 $\gamma$ further from the PDE6 $\gamma$ binding sites on the catalytic subunits and making these sites more easily accessible. One implication of these considerations is that the $\mathrm{T}_{\alpha}-\mathrm{GTP} / \mathrm{PDE} 6 \gamma$ complex must remain bound to the rest of the PDE6 heterotetramer until the terminal phosphate of the GTP has been hydrolyzed. If the $\mathrm{T}_{\alpha}$-GTP/ PDE6 $\gamma$ were to come entirely off the PDE6, as seems to occur in frog rods (Yamazaki, 1992), the rate of exogenous PDE6 $\gamma$ binding could not be affected by the presence or absence of RGS9-1 and the rest of the GAP proteins.

It is highly likely that excess PDE6 $\gamma$ turns off the cascade by binding directly to the PDE6 $\gamma$ binding sites on the PDE6 $\alpha$ and $\beta$ catalytic subunits rather than by binding to $\mathrm{T}_{\alpha}$-GTP and facilitating the removal of the G-protein from the PDE6 heterotetramer. The experiments of Otto-Bruc et al. (1993) show that the W70F form of PDE6 $\gamma$ binds much more weakly to $\mathrm{T}_{\alpha}-\mathrm{GTP} \gamma \mathrm{S}$ than does WT PDE6 $\gamma$, but both forms of PDE6 $\gamma$ inhibit activated PDE6 with similar efficiency. We propose that after the binding of exogenous PDE6 $\gamma$ to the catalytic site of activated PDE6, the complex of $\mathrm{T}_{\alpha}$-GTP and endogenous PDE6 $\gamma$ remains attached to the PDE6 heterotetramer, but after hydrolysis of $\mathrm{T}_{\alpha}$-GTP to $\mathrm{T}_{\alpha^{-}}$ GDP, the PDE6 $\gamma / \mathrm{T}_{\alpha}$-GDP complex dissociates and is released to the cytosol, because PDE6 $\gamma$ binds much less tightly to $\mathrm{T}_{\alpha}$-GDP than to $\mathrm{T}_{\alpha}$-GTP (Otto-Bruc et al., 1993). This would recycle endogenous PDE6 $\gamma$ to the pool of free inhibitor.

We summarize our conclusions in the schema of supplemental Figure 4 (available at www.jneurosci.org as supplemental material). In wt6C mouse rods, the $\mathrm{T}_{\alpha}$-GTP produced by light stimulation (top panel) may bind either directly to excess PDE6 $\gamma(\mathrm{A})$, reducing the gain of the light response, or to endogenous PDE6 $\gamma$, as in the WT rod (1), displacing the endogenous PDE6 $\gamma$ from its inhibitory binding sites and activating the PDE6. The $\mathrm{T}_{\alpha}$-GTP and endogenous PDE6 $\gamma$ then bind to RGS9-1 and its associated proteins (2), to form a complex that remains attached to the PDE6 heterotetramer.

The activity of the PDE6 can then be quenched in two ways 
(bottom panel). The RGS9-1 complex can accelerate the rate of hydrolysis of $\mathrm{T}_{\alpha}$-GTP to $\mathrm{T}_{\alpha}$-GDP (3), causing the $\mathrm{T}_{\alpha}$-GDP and RGS9-1 to come off the PDE6 and the PDE6 $\gamma$ to return to its sites on the PDE6 catalytic $\alpha$ or $\beta$ subunits. Alternatively, excess PDE6 $\gamma$ can bind directly to sites on the PDE6 catalytic $\alpha$ or $\beta$ subunits (B). We then suppose that once the $\mathrm{T}_{\alpha}$-GTP is hydrolyzed to $\mathrm{T}_{\alpha}$-GDP, the $\mathrm{T}_{\alpha}$-GDP and RGS9-1 fall off the PDE6, and the associated PDE6 $\gamma$ is also released back into the cytoplasmic pool of PDE6 $\gamma$. The postulated exchange of PDE6 $\gamma$ may also occur to some extent in WT mice, and this may explain the decay of rod photoresponses even in the presence of nonhydrolyzable analogs of GTP (Erickson et al., 1992).

Defects in RGS9-1 or R9AP in humans cause a condition that has been termed "bradyopsia," characterized by subnormal acuity, photophobia, and slow adaptation that produces a transient but debilitating blindness (Nishiguchi et al., 2004). The results in Figures 6 and 7 show that overexpression of PDE6 $\gamma$ has an epistatic effect on rods lacking the GAP complex, both for responses to flashes and for recovery after bright-light exposure. The responses of rods in $\mathrm{Rgs}_{\mathrm{s}} \mathrm{I}^{-/-}$mice in which PDE6 $\gamma$ has been overexpressed, although slower to recover from light exposure than WT rods, are nevertheless much more similar to WT responses that those of the rods of $R g s 9^{-/-}$mice without PDE6 $\gamma$ overexpression. We predict that the bradyopsia of patients lacking the GAP complex would be substantially ameliorated by PDE6 $\gamma$ overexpression in rod and cone photoreceptors. Because RGS9-1 and the other components of the GAP complex are expressed in many parts of the nervous system but the PDE6 $\gamma$ protein is expressed only in the photoreceptors, future pharmacological upregulation of PDE6 $\gamma$ may provide a safer and more easily implemented cure for bradyopsia and related conditions.

\section{References}

Angleson J, Wensel T (1993) A GTPase-accelerating factor for transducin, distinct from its effector cGMP phosphodiesterase, in rod outer segment membranes. Neuron 11:939-949.

Angleson J, Wensel T (1994) Enhancement of rod outer segment GTPase accelerating protein activity by the inhibitory subunit of cGMP phosphodiesterase. J Biol Chem 269:16290-16296.

Antonny B, Otto-Bruc A, Chabre M, Vuong TM (1993) GTP hydrolysis by purified alpha-subunit of transducin and its complex with the cyclic GMP phosphodiesterase inhibitor. Biochemistry 32:8646-8653.

Arshavsky V, Bownds MD (1992) Regulation of deactivation of photoreceptor $\mathrm{G}$ protein by its target enzyme and cGMP. Nature 357:416-417.

Arshavsky VY, Dumke CL, Zhu Y, Artemyev NO, Skiba NP, Hamm HE, Bownds MD (1994) Regulation of transducin GTPase activity in bovine rod outer segments. J Biol Chem 269:19882-19887.

Arshavsky VY, Lamb TD, Pugh Jr EN (2002) G proteins and phototransduction. Annu Rev Physiol 64:153-187.

Baylor DA, Matthews G, Yau KW (1980) Two components of electrical dark noise in toad retinal rod outer segments. J Physiol (Lond) 309:591-621.

Baylor DA, Nunn BJ, Schnapf JL (1984) The photocurrent, noise and spectral sensitivity of rods of the monkey Macaca fascicularis. J Physiol (Lond) 357:575-607.

Bondarenko VA, Yamazaki M, Hayashi F, Yamazaki A (1999) Suppression of GTP/T alpha-dependent activation of cGMP phosphodiesterase by ADP-ribosylation by its gamma subunit in amphibian rod photoreceptor membranes. Biochemistry 38:7755-7763.

Brann MR, Cohen LV (1987) Diurnal expression of transducin mRNA and translocation of transducin in rods of rat retina. Science 235:585-587.

Chen CK, Burns ME, He W, Wensel TG, Baylor DA, Simon MI (2000) Slowed recovery of rod photoresponse in mice lacking the GTPase accelerating protein RGS9-1. Nature 403:557-560.

Erickson MA, Robinson P, Lisman J (1992) Deactivation of visual transduction without guanosine triphosphate hydrolysis by $G$ protein. Science 257:1255-1258.
Fain GL (2003) Sensory transduction. Sunderland, MA: Sinauer.

Field GD, Rieke F (2002) Nonlinear signal transfer from mouse rods to bipolar cells and implications for visual sensitivity. Neuron 34:773-785.

Hamm HE, Bownds MD (1986) Protein complement of rod outer segments of frog retina. Biochemistry 25:4512-4523.

He W, Cowan CW, Wensel TG (1998) RGS9, a GTPase accelerator for phototransduction. Neuron 20:95-102.

Hu G, Wensel TG (2002) R9AP, a membrane anchor for the photoreceptor GTPase accelerating protein, RGS9-1. Proc Natl Acad Sci USA 99:9755-9760.

Keresztes G, Martemyanov KA, Krispel CM, Mutai H, Yoo PJ, Maison SF, Burns ME, Arshavsky VY, Heller S (2004) Absence of the RGS9.Gbeta5 GTPase-activating complex in photoreceptors of the R9AP knockout mouse. J Biol Chem 279:1581-1584.

Krispel CM, Chen CK, Simon MI, Burns ME (2003) Prolonged photoresponses and defective adaptation in rods of $\mathrm{G} \beta 5-/-$ mice. J Neurosci 23:6965-6971.

Krispel CM, Chen CK, Chen D, Chen YK, Calero N, Burns ME (2005) The rate limiting step in the deactivation of the phototransduction cascade. Invest Ophthalmol Vis Sci 46:ARVO E-Abstract (4626).

Lem J, Applebury ML, Falk JD, Flannery JG, Simon MI (1991) Tissuespecific and developmental regulation of rod opsin chimeric genes in transgenic mice. Neuron 6:201-210.

Leskov IB, Klenchin VA, Handy JW, Whitlock GG, Govardovskii VI, Bownds MD, Lamb TD, Pugh Jr EN, Arshavsky VY (2000) The gain of rod phototransduction: reconciliation of biochemical and electrophysiological measurements. Neuron 27:525-537.

Makino ER, Handy JW, Li T, Arshavsky VY (1999) The GTPase activating factor for transducin in rod photoreceptors is the complex between RGS9 and type 5 G protein beta subunit. Proc Natl Acad Sci USA 96:1947-1952.

Mendez A, Lem J, Simon M, Chen J (2003) Light-dependent translocation of arrestin in the absence of rhodopsin phosphorylation and transducin signaling. J Neurosci 23:3124-3129.

Nair KS, Hanson SM, Mendez A, Gurevich EV, Kennedy MJ, Shestopalov VI, Vishnivetskiy SA, Chen J, Hurley JB, Gurevich VV, Slepak VZ (2005) Light-dependent redistribution of arrestin in vertebrate rods is an energyindependent process governed by protein-protein interactions. Neuron 46:555-567.

Nishiguchi KM, Sandberg MA, Kooijman AC, Martemyanov KA, Pott JW, Hagstrom SA, Arshavsky VY, Berson EL, Dryja TP (2004) Defects in RGS9 or its anchor protein R9AP in patients with slow photoreceptor deactivation. Nature 427:75-78.

Otto-Bruc A, Antonny B, Vuong TM, Chardin P, Chabre M (1993) Interaction between the retinal cyclic GMP phosphodiesterase inhibitor and transducin. Kinetics and affinity studies. Biochemistry 32:8636-8645.

Pepperberg DR, Cornwall MC, Kahlert M, Hofmann KP, Jin J, Jones GJ, Ripps H (1992) Light-dependent delay in the falling phase of the retinal rod photoresponse. Vis Neurosci 8:9-18.

Philp NJ, Chang W, Long K (1987) Light-stimulated protein movement in rod photoreceptor cells of the rat retina. FEBS Lett 225:127-132.

Piriev NI, Yamashita C, Samuel G, Farber DB (1993) Rod photoreceptor cGMP-phosphodiesterase: analysis of a and b subunits expressed in human kidney cells. Proc Natl Acad Sci USA 90:9340-9344.

Pittler SJ, Baehr W (1991) Identification of a nonsense mutation in the rod photoreceptor cGMP phosphodiesterase $\beta$-subunit gene of the rd mouse. Proc Natl Acad Sci USA 88:8322-8326.

Rieke F, Baylor DA (1996) Molecular origin of continuous dark noise in rod photoreceptors. Biophys J 71:2553-2572.

Sagoo MS, Lagnado L (1997) G-protein deactivation is rate-limiting for shut-off of the phototransduction cascade. Nature 389:392-395.

Skiba NP, Hopp JA, Arshavsky VY (2000) The effector enzyme regulates the duration of $\mathrm{G}$ protein signaling in vertebrate photoreceptors by increasing the affinity between transducin and RGS protein. J Biol Chem 275:32716-32720.

Sokolov M, Lyubarsky AL, Strissel KJ, Savchenko AB, Govardovskii VI, Pugh EN Jr, Arshavsky VY (2002) Massive light-driven translocation of transducin between the two major compartments of rod cells: a novel mechanism of light adaptation. Neuron 34:95-106. 
Towbin H, Staehelin T, Gordon J (1979) Electrophoretic transfer of proteins from polyacrylamide gels to nitrocellulose sheets: procedure and some applications. Proc Natl Acad Sci USA 76:4350-4354.

Tsang SH, Gouras P, Yamashita CK, Kjeldbye H, Fisher J, Farber DB, Goff SP (1996) Retinal degeneration in mice lacking the gamma subunit of the rod cGMP phosphodiesterase. Science 272:1026-1029.

Tsang SH, Burns ME, Calvert PD, Gouras P, Baylor DA, Goff SP, Arshavsky VY (1998) Role of the target enzyme in deactivation of photoreceptor $G$ protein in vivo. Science 282:117-121.

Tsang SH, Woodruff ML, Lee W-H, Yamashita CY, Goff SP, Farber DB, Fain GL (2004) Regulation of photoreceptor signal termination. Invest Ophthalmol Vis Sci 45:ARVO E-Abstract (2206).

Wensel TG, Stryer L (1990) Activation mechanism of retinal rod cyclic GMP phosphodiesterase probed by fluorescein-labeled inhibitory subunit. Biochemistry 29:2155-2161.

Whelan JP, McGinnis JF (1988) Light-dependent subcellular movement of photoreceptor proteins. J Neurosci Res 20:263-270.

Woodruff ML, Sampath AP, Matthews HR, Krasnoperova NV, Lem J, Fain
GL (2002) Measurement of cytoplasmic calcium concentration in the rods of wild- type and transducin knock-out mice. J Physiol (Lond) 542:843-854

Woodruff ML, Wang Z, Chung HY, Redmond TM, Fain GL, Lem J (2003) Spontaneous activity of opsin apoprotein is a cause of Leber congenital amaurosis. Nat Genet 35:158-164.

Yamazaki A (1992) The GTP-binding protein-dependent activation and deactivation of cyclic GMP phosphodiesterase in rod photoreceptors. Adv Second Messenger Phosphoprotein Res 25:135-145.

Yamazaki A, Moskvin O, Yamazaki RK (2002) Phosphorylation by cyclindependent protein kinase 5 of the regulatory subunit (Pgamma) of retinal cgmp phosphodiesterase (PDE6): its implications in phototransduction. Adv Exp Med Biol 514:131-153.

Zhang H, Huang W, Zhu X, Craft CM, Baehr W, Chen CK (2003) Lightdependent redistribution of visual arrestins and transducin subunits in mice with defective phototransduction. Mol Vis 9:231-237.

Zimmerman WF, Godchaux WD (1982) Preparation and characterization of sealed bovine rod cell outer segments. Methods Enzymol 81:52-57. 